\title{
Forces Acting on a Biped Robot. Center of Pressure-Zero Moment Point
}

\author{
Philippe Sardain and Guy Bessonnet
}

\begin{abstract}
In the area of biped robot research, much progress has been made in the past few years. However, some difficulties remain to be dealt with, particularly about the implementation of fast and dynamic walking gaits, in other words anthropomorphic gaits, especially on uneven terrain. In this perspective, both concepts of center of pressure (CoP) and zero moment point (ZMP) are obviously useful. In this paper, the two concepts are strictly defined, the CoP with respect to ground-feet contact forces, the ZMP with respect to gravity plus inertia forces. Then, the coincidence of $\mathrm{CoP}$ and $\mathrm{ZMP}$ is proven, and related control aspects are examined. Finally, a virtual CoP-ZMP is defined, allowing us to extend the concept when walking on uneven terrain. This paper is a theoretical study. Experimental results are presented in a companion paper, analyzing the evolution of the ground contact forces obtained from a human walker wearing robot feet as shoes.
\end{abstract}

Index Terms-Biped robot, center of pressure (CoP), mechanical feet, uneven terrain, walking, zero moment point (ZMP).

\section{INTRODUCTION}

$\mathbf{T}$ WO FRENCH laboratories, LMS and INRIA RhôneAlpes, have designed and constructed an anthropomorphic biped robot, Bip. The goals and initial results of the project are reported in [1] and [2] and the implementation of the postural motions and static walks achieved until now are described in [3]. The current research is directed toward the generation of anthropomorphic trajectories, and toward efficient ways for the biped robot to control them. The robot is fitted with feet equipped with sensors measuring the ground-foot forces, in order to exploit the concepts of center of pressure $(\mathrm{CoP})$ and zero moment point (ZMP). The notion of ZMP has been known about for more than thirty years, but is in no way old-fashioned, since as long as gravity forces govern walking gaits, the ZMP will be a significant dynamic equilibrium criterion. In addition, this quite useful concept has not been completely explored, and unfortunately some misinterpretations are sometimes encountered in the literature.

In this paper, we refer the point associated with contact forces as CoP, while ZMP is considered to be related to gravity plus inertia forces. Strict definitions of CoP and ZMP are specified in Section II, and concise algebraic relationships for the computation of both points are formulated. In Section III, before

Manuscript received December 11, 2002; revised February 25, 2004. This work was supported in part by the Council of the Poitou-Charentes Region and the Council of the Vienne Department, France, under Grant 95/RPC-R-94. This paper was recommended by Associate Editor C. E. Smith.

The authors are with the LMS, Laboratoire de Mécanique des Solides, CNRSUniversité de Poitiers, BP 30179, 86962 Futuroscope Chasseneuil Cedex, France (e-mail: Philippe.Sardain@1ms.univ-poitiers.fr; Guy.Bessonnet@1ms. univ-poitiers.fr).

Digital Object Identifier 10.1109/TSMCA.2004.832811 discussing the relevance of the CoP-ZMP as a means of controlling the dynamic equilibrium of bipeds, the coincidence of the two points is proven. Finally, Section IV suggests an extension of the CoP-ZMP concept when the feet of the biped do not lie in the same plane. Indeed, the concept holds only with respect to a single plane, and cannot be directly applied when the biped walks across an uneven terrain. The understanding of the demonstrations on the one hand, and the physical interpretation of the phenomena on the other hand are facilitated by the notion of the central axis of moment field, which is recalled in Appendix I.

This theoretical study is aimed at clarifying and extending the CoP-ZMP concepts. As a perspective, to go further toward the knowledge of walking gaits with mechanical feet, specific data are required. The tests carried out to validate the instrumentation of the Bip feet have been performed by a human walker wearing the Bip rigid soles as shoes [4]. This experimental approach has proved to be a relevant way of studying the influence of "mechanical" feet on the gait of the subject. The results are presented and analyzed in the companion paper [5].

\section{COP VERSUS ZMP}

The concepts of CoP and ZMP are quite useful for the control of the dynamic equilibrium of bipeds, but first the exact meaning we attach to these notions has to be defined.

\section{A. Definitions}

The forces acting on a walker can be separated in two categories: 1) forces exerted by contact and 2) forces transmitted without contact (gravity and, by extension, inertia forces). The $\mathrm{CoP}$ is linked to the former, and the ZMP to the latter.

1) CoP Definition: Let us consider a biped when one single foot is in contact with the ground. The field of pressure forces (normal to the sole) is equivalent to a single resultant force, exerted at the point where the resultant moment is zero. This point is termed CoP.

At any point $P$ of the sole [see Fig. 1(a)], the elementary contact force $\boldsymbol{c}(P)$ can be split up into two components, a normal component $p(P) \boldsymbol{n}$, which is the local pressure force, and a tangential component $f(P)$ which is the local frictional force

$$
\begin{gathered}
P \in(S), \quad \boldsymbol{c}(P)=p(P) \boldsymbol{n}+\boldsymbol{f}(P) \\
p(P) \geq 0, \quad \boldsymbol{f}(P) \cdot \boldsymbol{n}=\mathbf{0} .
\end{gathered}
$$

with $\boldsymbol{n}$ being the unit vector normal to the sole $(S)$, oriented toward the foot, outwards from the support surface. 


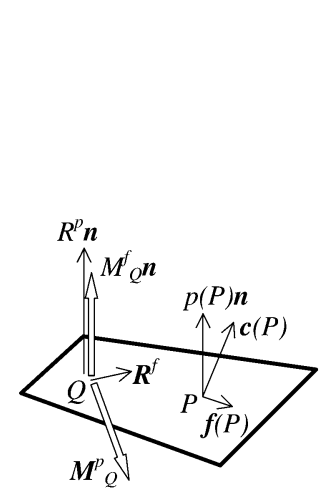

(a)

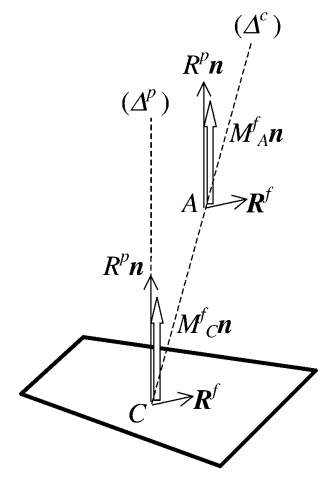

(b)
Fig. 1. Contact forces and moments acting on the sole.

Consequently, the resultant of these elementary contact forces appears as the sum, on the one hand, of the local pressure forces

$$
\boldsymbol{R}^{p}=\left(\int_{S} p(P) d S\right) \boldsymbol{n} \equiv R^{p} \boldsymbol{n}, \quad R^{p}>0
$$

whose moment about any point $Q$ is

$$
\boldsymbol{M}_{Q}^{p}=\left(\int_{S} p(P) \boldsymbol{Q P} d S\right) \times \boldsymbol{n}
$$

and, on the other hand, of the local friction forces

$$
\boldsymbol{R}^{f}=\int_{S} \boldsymbol{f}(P) d S, \quad \boldsymbol{R}^{f} \cdot \boldsymbol{n}=0
$$

whose moment about $Q$ is

$$
\boldsymbol{M}_{Q}^{f}=\int_{S} \boldsymbol{Q P} \times \boldsymbol{f}(P) d S
$$

with the superscripts $p$ and $f$ denoting pressure and friction components, respectively.

Relationship (3) shows that the moment of the pressure forces is always perpendicular to the normal vector $\boldsymbol{n}$, whatever the point $Q$. Consequently, as on the other hand, the resultant of the pressure forces is directed along $\boldsymbol{n}$, then one axis $\left(\Delta^{p}\right)$ exists, where the moment $\boldsymbol{M}_{Q}^{p}$ vanishes at every point of this axis. One can notice that $\left(\Delta^{p}\right)$ is the central axis of the pressure force wrench, as defined in Appendix I-A. The CoP $C$ which is defined as the point of the sole where $M_{C}^{p}=\mathbf{0}$ is the intersection between the axis $\left(\Delta^{p}\right)$ and the plane of the sole [see Fig. 1(b)].

Relationship (5) shows that the moment of the friction forces is parallel to the normal vector $\boldsymbol{n}$ if the point $Q$ belongs to the plane of the sole. This is true at the level of $C$, because the CoP is a point on the sole. Therefore, the $\operatorname{CoP} C$ can also be defined as the point on the sole where the moment of the contact forces is perpendicular to the sole

$$
\begin{aligned}
\boldsymbol{R}^{c} & =R^{p} \boldsymbol{n}+\boldsymbol{R}^{f}, \quad R^{p}>0, \quad \boldsymbol{R}^{f} \cdot \boldsymbol{n}=0 \\
\boldsymbol{M}_{C}^{c} & =\boldsymbol{M}_{C}^{f} \boldsymbol{n}
\end{aligned}
$$

where the superscript $c$ denotes contact force and moment.
Considering the contact forces, i.e., pressure plus friction forces, one axis $\left(\Delta^{c}\right)$ exists where the moment $\boldsymbol{M}_{A}^{c}$ at every point $A$ of this axis is parallel to $\boldsymbol{n}$ (and $M_{A}^{c}=M_{C}^{f} \boldsymbol{n}$ ). The CoP $C$ can finally be defined as the intersection between the axis $\left(\Delta^{c}\right)$ and the plane of the sole [see Fig. 1(b)]. One can remark that $\left(\Delta^{c}\right)$ is a noncentral axis of the contact force wrench, as defined in Appendix I-A.

It should be noted that if the two feet are in contact with a plane ground, then all previous assumptions hold. The area $(S)$ to be considered in order to define the $\mathrm{CoP}$ is the total area where contact forces appear. The $\mathrm{CoP}$ is a point of the supporting plane of the two feet, it falls within the convex hull of the two contact areas.

2) ZMP Definition: The ZMP is the point on the ground where the tipping moment acting on the biped, due to gravity and inertia forces, equals zero, the tipping moment being defined as the component of the moment that is tangential to the supporting surface.

The ZMP concept was introduced and developed in [6] and [7]. It has been exhaustively reviewed in [8]. It is a key point in the control of the Honda biped robots [9]. The Honda Co. has patented many detailed implementation developments [10]-[12].

It should be noted that the term ZMP is not a perfectly exact expression because the normal component of the moment generated by the inertia forces acting on the biped is not necessarily zero. If we bear in mind, however, that ZMP abridges the exact expression "zero tipping moment point," then the term becomes perfectly acceptable.

The resultant of the gravity plus inertia forces (superscript $g i$ ) may be expressed as

$$
\boldsymbol{R}^{g i}=m \boldsymbol{g}-m \boldsymbol{a}_{G}
$$

and the moment about any point $Q$ as

$$
\boldsymbol{M}_{Q}^{g i}=Q \boldsymbol{G} \times m \boldsymbol{g}-Q \boldsymbol{G} \times m \boldsymbol{a}_{G}-\dot{\boldsymbol{H}}_{G}
$$

where $m$ is the total mass, $\boldsymbol{g}$ is the acceleration of the gravity, $G$ is the center of mass (CoM) of the biped, $\boldsymbol{a}_{G}$ is the acceleration of $G$, and $\dot{\boldsymbol{H}}_{G}$ is the rate of angular momentum at $G$. More developed expressions of (8) and (9) are proposed in Appendix II-A.

One axis $\left(\Delta^{g i}\right)$ exists, where the moment is parallel to the normal vector $\boldsymbol{n}$, about every point $B$ of the axis. The ZMP, "zero tipping moment point," whose moment is by definition directed along the normal vector $\boldsymbol{n}$, necessarily belongs to this axis. So, $D$, the ZMP, can be defined as the intersection between the axis $\left(\Delta^{g i}\right)$ and the ground surface (see Fig. 2), such that

$$
\boldsymbol{M}_{D}^{g i}=\boldsymbol{D} \boldsymbol{G} \times m \boldsymbol{g}-\boldsymbol{D} \boldsymbol{G} \times m \boldsymbol{a}_{G}-\dot{\boldsymbol{H}}_{G}, \quad \boldsymbol{M}_{D}^{g i} \times \boldsymbol{n}=\mathbf{0} .
$$

It should be noted that this particular axis does not pass through the global CoM $G$, although some authors draw as such in their diagrams (as in [8] and [9] ), and although some stability criteria are based on the position of the projection of the CoM along $\boldsymbol{R}^{g i}$ (see [13] for a bibliography). The position of the axis $\left(\Delta^{g i}\right)$ - and by extension $\left(\Delta^{c}\right)$ - is clearly established in Appendix I-B. 


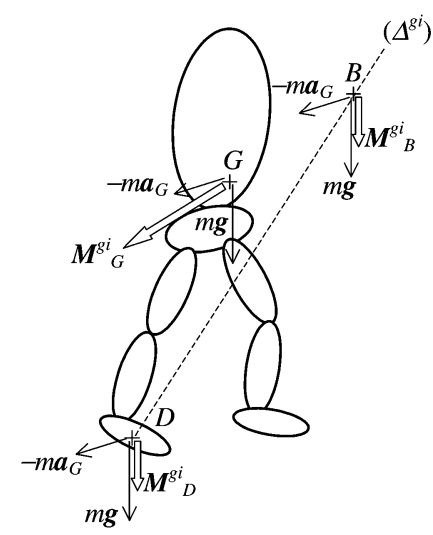

Fig. 2. Gravity-inertia forces-moments at the CoM $G$, and the same transported at the ZMP $D$.

\section{B. Expressions of the CoP and ZMP}

The CoP and the ZMP, as defined above, can be computed as follows.

1) $\mathrm{CoP}$ Computation: Let us consider $O$, a point of the sole (generally the normal projection of the ankle), and $\boldsymbol{n}$, the unit normal vector directed outwards from the support surface.

Knowing the expression of the pressure forces about the point $O$, in other words knowing $\left\{\boldsymbol{R}^{p}, \boldsymbol{M}_{O}^{p}\right\}$, the problem consists in determining the position $C$ of the CoP.

By definition, $C$ is the point where the moment of the pressure forces vanishes. Therefore,

$$
\boldsymbol{M}_{O}^{p}=O C \times \boldsymbol{R}^{p}
$$

and consequently the vector $O C$ can be expressed as

$$
O \boldsymbol{C}=\frac{\boldsymbol{n} \times M_{O}^{p}}{R^{p}} .
$$

It is straightforward to establish that $O C$ can be formulated as the function

$$
\boldsymbol{O C}=\frac{\boldsymbol{n} \times M_{O}^{c}}{\boldsymbol{R}^{c} \cdot \boldsymbol{n}}
$$

of the total contact forces $\left\{\boldsymbol{R}^{c}, \boldsymbol{M}_{O}^{c}\right\}$ about the point $O$.

2) ZMP Computation: The Newton-Euler equations of the global motion of the biped can be written as

$$
\begin{aligned}
\boldsymbol{R}^{c}+m \boldsymbol{g} & =m \boldsymbol{a}_{G} \\
\boldsymbol{M}_{Q}^{c}+\boldsymbol{Q} \boldsymbol{G} \times m \boldsymbol{g} & =\dot{\boldsymbol{H}}_{G}+\boldsymbol{Q} \boldsymbol{G} \times m \boldsymbol{a}_{G}
\end{aligned}
$$

and considered under the form

$$
\begin{array}{r}
\boldsymbol{R}^{c}+\left(m \boldsymbol{g}-m \boldsymbol{a}_{G}\right)=\mathbf{0} \\
M_{Q}^{c}+\left(\boldsymbol{Q} \boldsymbol{G} \times m \boldsymbol{g}-\boldsymbol{Q G} \times m \boldsymbol{a}_{G}-\dot{\boldsymbol{H}}_{G}\right)=\mathbf{0}
\end{array}
$$

to put together the gravity plus inertia forces so that (14) and (15) may be rewritten as

$$
\begin{aligned}
\boldsymbol{R}^{c}+\boldsymbol{R}^{g i} & =\mathbf{0} \\
\boldsymbol{M}_{Q}^{c}+\boldsymbol{M}_{Q}^{g i} & =\mathbf{0} .
\end{aligned}
$$

These equations express the fact that the biped is dynamically balanced if the contact forces and the gravity-inertia forces are strictly opposite.

Because of the opposition between the gravity-inertia forces and the contact forces, the ZMP $D$ is defined by an expression similar to (13)

$$
\boldsymbol{O D}=\frac{\boldsymbol{n} \times \boldsymbol{M}_{O}^{g i}}{\boldsymbol{R}^{g i} \cdot \boldsymbol{n}} .
$$

The widely favored formulations often met in literature, which one can sum up as

$$
\boldsymbol{O D}=\frac{m g \boldsymbol{z} \times \boldsymbol{O} \boldsymbol{G} \times \boldsymbol{z}+\boldsymbol{z} \times \dot{\boldsymbol{H}}_{O}}{m g+m \boldsymbol{a}_{G} \cdot \boldsymbol{z}}
$$

are only true if the ground is horizontal, i.e., if $\boldsymbol{n}=\boldsymbol{z}$ with $g=-g \boldsymbol{z}$. Else, if the contact surface is inclined, then (20) has to be used. Developments are given in Appendix II-B.

\section{COP AND ZMP COINCIDENCE}

The fact that the two points coincide has some importance: on the one hand for the understanding of the dynamic equilibrium of walking gaits, on the other hand for the control of walking robots.

\section{A. Demonstration of the Coincidence}

Because of (18) and (19), it is obvious that the axes $\left(\Delta^{c}\right)$ and $\left(\Delta^{g i}\right)$ coincide (indeed, they are noncentral axes defined from two opposite wrenches). We have shown that the CoP and the ZMP are the intersections of these axes with the supporting plane ground surface. Therefore, the CoP and the ZMP are the same point, called $C$, and obviously, as for any point $Q$ of the supporting plane ground surface, one gets

$$
M_{C}^{c}+M_{C}^{g i}=\mathbf{0} .
$$

This fact does not admit any discussion, it is true whatever the stability of the balance is, and in particular it is true when the walker is falling down, as long as a contact exists with the ground. Nevertheless, this evidence is sometimes discussed in the case of the fall [14], and that provokes some misinterpretations. When the CoP reaches the edge of the support polygon, the system as a whole rotates about the foot edge. In this case, the system becomes under-actuated. Yet, (18) and (19) represent the (trivial and obvious) proof that $\mathrm{CoP}$ and ZMP coincide.

A difficulty appears when the two feet are lying on two distinct surfaces. Indeed, the concept of CoP and that of ZMP use in their fundamental definitions the notion of vector normal to the contact surface and the notion of intersection between an axis and this surface. So the concepts are intrinsically related to walking on a single plane surface (whatever the form of the contacts, flat or no, for instance the rear foot can be heel-off and the front foot heel-strike). Consequently, the definitions are unfounded, for the double support phase, if the biped feet are contacting two noncoincident surfaces of an uneven terrain. Section IV proposes in this case the notions of virtual equivalent surface and pseudo-ZMP. Then, a pseudo-CoP exists, and the fundamental principles of dynamics, expressed by (18) and (19), lead again to the fact that pseudo-CoP and pseudo-ZMP coincide. 


\section{B. Interpretation of the Coincidence}

With regard of our definitions, the coincidence of CoP and ZMP is not surprising since they are two interpretations of acting force-moment between the ground and the first link of a kinematic chain. One interpretation (ZMP) is related to the accumulated inertia and gravity force-moment of the chain. The other $(\mathrm{CoP})$ is linked to the ground reaction force-moment, which equilibrates with the accumulated one. The same understanding is repeatedly used in dynamics computation such as the Newton-Euler recursive inverse dynamics (in the case of opened kinematic chains).

So, to conclude this section, in order to delete the misinterpretations appearing in literature and subsisting in some minds, we can say that, as long as all the ground-sole contacts appear in a single plane surface, then the CoP and the ZMP are absolutely and definitely the same point, that consequently we call CoP-ZMP. In case of two noncoincident contact surfaces, if one defines a virtual equivalent surface, then the corresponding pseudo-CoP and pseudo-ZMP are still the same point.

\section{Control Aspects}

The unilaterality of the foot-ground contact is a major constraint of legged locomotion. The fact that the pressure force is oriented toward the foot, outwards from the support surface, implies that the CoP lies within the support polygon (convex hull of the contact points or surfaces). Because of the CoP and ZMP coincidence, the latter, assumed to be linked to the gravity-inertia forces, is submitted to the same constraint.

Of course, when the biped is flying, the support polygon disappears, and consequently the CoP-ZMP is not defined. A contrario, the CoP-ZMP can be used as a control criterion when a support area exists. This is precisely the definition of walking (a support area always exists), and thus the CoP-ZMP criterion is perfectly relevant for characterizing the tipping equilibrium of walking bipeds.

The major advantage of the CoP-ZMP concept is that this point can be measured: measuring the contact pressure forcemoment allows the CoP to be reconstructed, and the ZMP by coincidence, and therefore the corresponding part of the gravityinertia forces. The CoP-ZMP concept has been used explicitly by several authors, and implicitly by many others, those who directly monitor the contact forces [15].

For a complete bibliography, see [8] and [13]. Of the earlier works related to control carried out with the ZMP concept, only one is referred to here: in the Honda biped robots, an application of the CoP-ZMP control has been implemented, showing that the CoP notion is related to contact forces, and that of the ZMP to gravity plus inertia forces [9]. Indeed, the authors use two types of control, the first being assumed acting on the CoP (denoted as C-ATGR in their paper) by "lowering" the heel or the toe of the foot, the second being assumed acting on the ZMP by increasing the magnitude of the inertia forces (accelerating the trunk position). The distinction goes in a direction analog to our definitions of CoP and ZMP: the CoP (C-ATGR) is invoked when the actuation directly affects the foot (i.e., the contact forces), the ZMP when the trunk acceleration is modified (consequently the gravity plus inertia forces). What the authors

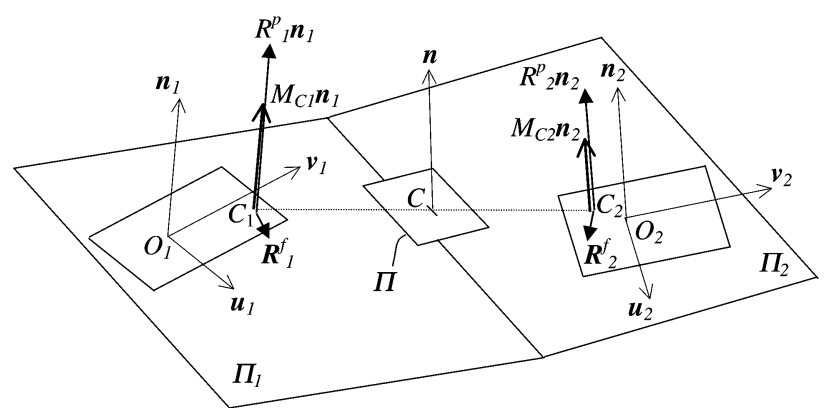

Fig. 3. The matter of two noncoincident contact surfaces, according to Honda US Patent no. 5357433.

do not say is that a modification of the CoP causes instantaneously a ZMP modification, and vice versa, because the two points coincide. An action about the ankle modifies the contact forces, certainly, but also the biped configuration. An acceleration of the trunk increases the inertia forces, certainly, but modifies the contact forces too. In other words, the modifications happen simultaneously.

\section{ADAPTATION OF THE COP-ZMP CONCEPT IN THE CASE OF UNEVEN TERRAIN}

The CoP and ZMP concepts use in their fundamental definitions the vector normal to the ground surface (for the definition of axes $\Delta^{c}$ and $\Delta^{g i}$ ), and the ground plane itself (which intersects the axes). So, the concepts are intrinsically related to walking on one single plane surface (associated to one single normal vector). The definitions are unsuitable, for the double support phase when the biped feet are contacting two noncoincident surfaces.

\section{A. Virtual Surface and Virtual CoP-ZMP}

The Honda US Patent [10] tackles the matter of irregular terrain by defining a virtual ZMP and a virtual surface varying continuously from the first to the second surface during the weight transfer from one foot to the other (see Fig. 3).

According to the authors, the virtual ZMP $C$ is a weighting function of the local ZMP's $C_{1}$ and $C_{2}$, such that

$$
\boldsymbol{O C}=(1-h) O \boldsymbol{C}_{1}+h O \boldsymbol{C}_{2}
$$

where $h$ is a function varying continuously from 0 to 1 . The authors have chosen a function proportional to the duration of the double support phase, $h=\left(t-t_{\text {start }}\right) /\left(t_{\text {end }}-t_{\text {start }}\right)$.

The virtual surface is defined by the ZMP $C$ and by the normal vector $\boldsymbol{n}$, resulting of an identical weighting function as that of $C$, such that

$$
\boldsymbol{n}=(1-h) \boldsymbol{n}_{1}+h \boldsymbol{n}_{2} .
$$

The idea is very good, because it provides the continuity of the contact forces (and consequently of the gravity-inertia ones) from the single support to the double one. However, a detailed study proves that these weighting functions are not in adequacy with the ZMP concept, requiring that the moment of the contact forces is perpendicular to the surface. Indeed, one can show that if the point $C$ is defined by (23), then the contact moment $M_{C}^{c}$ is not directed along $\boldsymbol{n}$ defined by (24). 


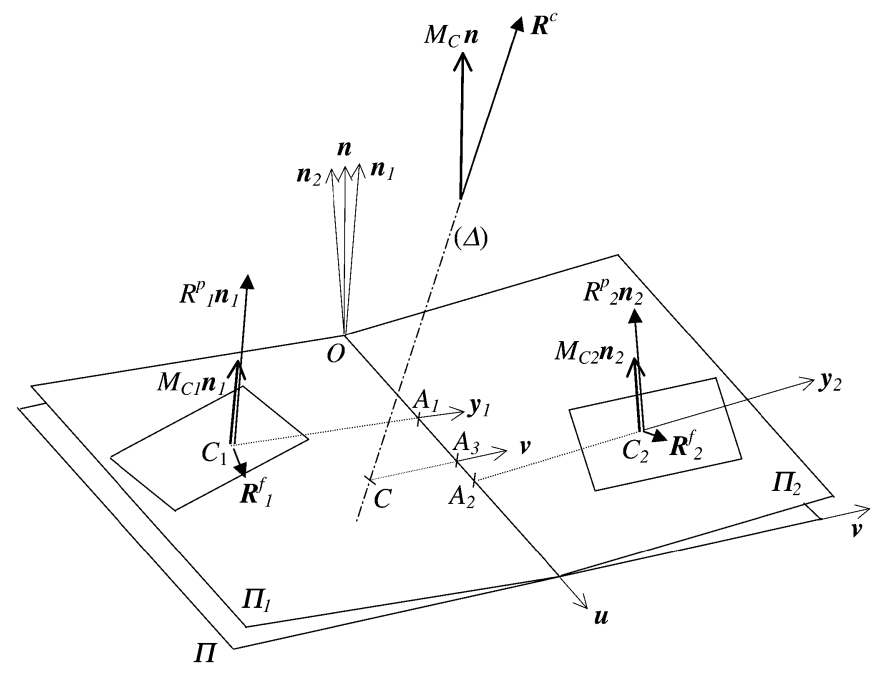

Fig. 4. Pseudo-CoP-ZMP $C$ and virtual surface $\Pi$ (proposition of this paper).

\section{B. Proposition Respecting the CoP-ZMP Concept}

We suggest to define a virtual surface $\Pi$ equivalent to the two real surfaces $\Pi_{1}$ and $\Pi_{2}$, then to chose the pseudo-CoP-ZMP lying in this surface such that the moment of the contact forces is perpendicular to $\Pi$.

When the surface $\Pi$ is defined (and consequently its normal vector $\boldsymbol{n})$, then an axis $(\Delta)$ exists such that the contact moment $\boldsymbol{M}_{P}^{c}$ is parallel with $\boldsymbol{n}$, whatever the points $P$ of $(\Delta)$ are (see demonstration in Appendix I). The pseudo-CoP-ZMP $C$ is the intersection point between $(\Delta)$ and $\Pi$, as Fig. 4 shows.

We suggest that the surface $\Pi$ passes through the intersection line of the two real surfaces $\Pi_{1}$ and $\Pi_{2}$, i.e., through line $(O, \boldsymbol{u})$, where

$$
\boldsymbol{u}=\frac{\boldsymbol{n}_{1} \times \boldsymbol{n}_{2}}{\left\|\boldsymbol{n}_{1} \times \boldsymbol{n}_{2}\right\|}
$$

and that its normal vector is defined as a weighting function of $\boldsymbol{n}_{1}$ and $\boldsymbol{n}_{2}$, the weight factors being respectively proportional to the local pressure contact forces $R_{1}^{p}$ and $R_{2}^{p}$, such that

$$
\boldsymbol{n}=\frac{R_{1}^{p} \boldsymbol{n}_{1}+R_{2}^{p} \boldsymbol{n}_{2}}{\left\|R_{1}^{p} \boldsymbol{n}_{1}+R_{2}^{p} \boldsymbol{n}_{2}\right\|} .
$$

The computation of the virtual CoP-ZMP $C$ is then carried out with

$$
\boldsymbol{O C}=\frac{\boldsymbol{n} \times M_{O}^{c}}{\boldsymbol{R}^{c} \cdot \boldsymbol{n}}
$$

where $\boldsymbol{R}^{c}$ and $\boldsymbol{M}_{O}^{c}$ are the resultant contact force and moment, such that

$$
\begin{aligned}
\boldsymbol{R}^{c} & =\boldsymbol{R}_{1}^{c}+\boldsymbol{R}_{2}^{c} \\
\boldsymbol{M}_{O}^{c} & =\boldsymbol{M}_{C_{1}}^{c}+O \boldsymbol{C}_{1} \times \boldsymbol{R}_{1}^{c}+\boldsymbol{M}_{C_{2}}^{c}+O C_{2} \times \boldsymbol{R}_{2}^{c}
\end{aligned}
$$

where $\boldsymbol{R}_{i}^{c}$ and $\boldsymbol{M}_{C_{i}}^{c}$ are the local contact force and moment at foot no. $i$.

A general expression of $\boldsymbol{O C}$ if proposed in Appendix III, where the weight factors are such that

$$
\boldsymbol{n}=\alpha_{1} \boldsymbol{n}_{1}+\alpha_{2} \boldsymbol{n}_{2}, \quad \text { where } \alpha_{2} \in[0,1] \text { and } \alpha_{1}=1-\alpha_{2}
$$

and where vector $O C$ is decomposed such that

$$
\boldsymbol{O C}=O A_{3} \boldsymbol{u}+A_{3} C \boldsymbol{v}
$$

One must notice that, whatever the weight factors $\alpha_{1}$ and $\alpha_{2}$ are, one neither gets

$$
O A_{3}=\alpha_{1} \cdot O A_{1}+\alpha_{2} \cdot O A_{2}
$$

nor

$$
A_{3} C=\alpha_{1} \cdot A_{1} C_{1}+\alpha_{2} \cdot A_{2} C_{2}
$$

The previous remark corroborates the assertion of Section IV-A, claiming that the simultaneous use of (23) and (24) is incompatible with the CoP-ZMP concept.

A contrario, the method we propose to define the virtual surface and the pseudo-ZMP is based on the definition of the CoPZMP. Moreover, the weight factors we suggest in (26) give good results, in accord with (32) and (33), as it will be discussed in Appendix III.

The generalization of the ZMP concept would be actually complete if we could defined what is the pseudosupport-polygon, a certain projection of the three-dimensional (3-D) convex hull (built from the two real support areas) onto the virtual surface $\Pi$, inside which the pseudo-ZMP stays. The ideas developed in [16] are very clever and seem to be a good inspiration source for a research in this direction. Nevertheless, we did not achieve an exact demonstration, considering the unilaterality of contacts on the one hand, and the friction on the other hand. This question is not crucial if the angle between the two planes $\Pi_{1}$ and $\Pi_{2}$ is not too large, as the experiments presented in paper [5] will show. A contrario, if the two planes are very angled, then it is in our mind necessary to monitor the whole contact forces, as pointed out in Section III-C (see [15]).

\section{Case of Noncoincident Parallel Surfaces (Stairs)}

In stairs, the feet are supported by parallel surfaces that have different elevations. During the double support phase, the virtual surface is naturally parallel to the others. The pseudo-CoP-ZMP $C$ is a weighting function of the local CoP-ZMP's $C_{1}$ and $C_{2}$, such that

$$
\alpha_{1} C \boldsymbol{C}_{1}+\alpha_{2} \boldsymbol{C} \boldsymbol{C}_{2}=\mathbf{0}
$$

where

$$
\alpha_{2} \in[0,1] ; \quad \alpha_{1}=1-\alpha_{2}
$$

In this case, whatever the weight factors are, the CoP-ZMP concept (moment of the contact forces perpendicular to the surface), is respected. From analogy with the previous case, a good choice is to take the weight factors proportional to the local pressure forces $R_{1}^{p}$ and $R_{2}^{p}$, such as

$$
\begin{aligned}
& \alpha_{1}=\frac{R_{1}^{p}}{\left(R_{1}^{p}+R_{2}^{p}\right)} \\
& \alpha_{2}=\frac{R_{2}^{p}}{\left(R_{1}^{p}+R_{2}^{p}\right)} .
\end{aligned}
$$




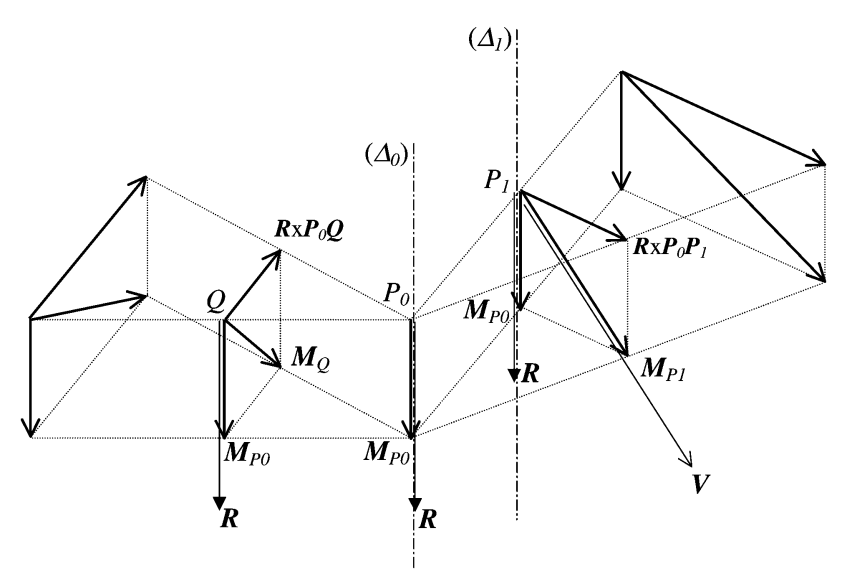

Fig. 5. Central and noncentral axes of a moment field: $\left(\Delta_{0}\right)=$ central axis ( $M_{P 0}$ and $R$ parallel), $\left(\Delta_{1}\right)=a$ noncentral axis (( $M_{P 1}$ and $R$ non-parallel).

\section{Conclusion AND Perspective}

The formal study presented in this paper has established strict definitions about the concepts of CoP and ZMP, clarifying certain misinterpretations sometimes encountered in the literature, and has proposed the concept of pseudo-CoP-ZMP related to walking on uneven terrain. As a perspective of this theoretical study, from a practical point of view, one must note that, among the numerous biped robots built world-wide, few, if any, are provided with anthropomorphic soles (the same is true in the case of Bip, our biped robot). What is the evolution of the CoP-ZMP for walking with such robot soles? Are these nonanthropomorphic soles compatible with human walking gaits? Experimental results answering to these questions are presented in the companion paper [5], as well as results linked to walking on uneven terrain and on stairs, showing the evolution of the pseudo-CoP-ZMP defined in this paper.

\section{APPENDIX I}

\section{A. Central and Noncentral Axes}

Let us consider a moment field, i.e., a space of points and a set of moments defined about every point $P$ from

$$
M_{P}=M_{Q}+R \times Q P
$$

where the invariant vector $\boldsymbol{R}$ and the moment $\boldsymbol{M}_{Q}$ are known.

One axis $\left(\Delta_{0}\right)$ exists (see Fig. 5), called Central Axis of the moment field, where the invariant vector $\boldsymbol{R}$ and the moment $M_{P_{0}}$ of the wrench are parallel, whatever the points $P_{0}$ belonging to $\left(\Delta_{0}\right)$ are. It is straightforward to prove that the central axis

$$
\left(\Delta_{0}\right)=\left\{P_{0} \text { such that } \boldsymbol{M}_{P_{0}} \times \boldsymbol{R}=\mathbf{0}\right\}
$$

is defined by

$$
\boldsymbol{Q} \boldsymbol{P}_{0}=\frac{\boldsymbol{R} \times \boldsymbol{M}_{Q}}{R^{2}}+\lambda \boldsymbol{R}, \quad \forall \lambda \in \Re .
$$

The central-axis concept can be expanded at points $\boldsymbol{P}_{1}$, which moments $\boldsymbol{M}_{P_{1}}$ are parallel with $\boldsymbol{V}$, whatever the vector $\boldsymbol{V}$ is. The noncentral axis

$$
\left(\Delta_{1}\right)=\left\{P_{1} \text { such that } \boldsymbol{M}_{P_{1}} \times \boldsymbol{V}=\mathbf{0}\right\}
$$

is defined by

$$
\begin{aligned}
& \boldsymbol{Q P} \boldsymbol{P}_{1}=\boldsymbol{Q} \boldsymbol{P}_{0}+\boldsymbol{P}_{0} \boldsymbol{P}_{1}: \\
& \boldsymbol{Q P}_{1}=\frac{\boldsymbol{R} \times \boldsymbol{M}_{Q}}{R^{2}}-\frac{\left(\boldsymbol{R} \cdot \boldsymbol{M}_{Q}\right) \boldsymbol{R} \times \boldsymbol{V}}{(\boldsymbol{R} \cdot \boldsymbol{V}) R^{2}}+\lambda \boldsymbol{R}, \quad \forall \lambda \in \Re .
\end{aligned}
$$

The central axis $\left(\Delta_{0}\right)$, such that $M_{P_{0}}$ and $R$ are parallel, and the noncentral axis $\left(\Delta_{1}\right)$, such that $\boldsymbol{M}_{P_{1}}$ is parallel to $\boldsymbol{V}$, are sketched in Fig. 5. Moreover, moments are sketched about points situated on the line $P_{0} Q$ and on the line $P_{0} P_{1}$. One can remark that all the moments in the space are distributed about the central axis $\left(\Delta_{0}\right)$ as the velocities resulting from a translation (" $\boldsymbol{M}_{P_{0}}$ ") plus a rotation (" $\boldsymbol{R}$ ") are. This is an explanation of the term "wrench" to refer to a moment field. From a similar point of view, a noncentral axis has a physical significance in the fact that all the points on $\left(\Delta_{1}\right)$-parallel to $\left(\Delta_{0}\right)$-have the same velocity (" $M_{P_{1}}$ ").

\section{B. Application to ZMP}

The gravity-inertia forces-moments are known about $G$, the biped CoM. The resultant force is

$$
\boldsymbol{R}^{g i}=m\left(\boldsymbol{g}-\boldsymbol{a}_{G}\right)
$$

and the resultant moment is

$$
\boldsymbol{M}_{G}^{g i}=-\dot{\boldsymbol{H}}_{G}
$$

The central axis

$$
\left(\Delta_{0}\right)=\left\{P_{0} \text { such that } \boldsymbol{M}_{P_{0}}^{g i} \times \boldsymbol{R}^{g i}=\mathbf{0}\right\}
$$

is defined by

$$
\boldsymbol{G} \boldsymbol{P}_{0}=\frac{\boldsymbol{R}^{g i} \times \boldsymbol{M}_{G}^{g i}}{R^{2}}+\lambda \boldsymbol{R}^{g i}, \quad \forall \lambda \in \Re .
$$

The axis

$$
\left(\Delta_{1}\right)=\left\{P_{1} \text { such that } \boldsymbol{M}_{P_{1}}^{g i} \times \boldsymbol{n}=\mathbf{0}\right\}
$$

where the moment of the gravity-inertia forces is directed along $\boldsymbol{n}$, the unit vector normal to the ground, is defined by

$$
\begin{aligned}
\boldsymbol{G} \boldsymbol{P}_{1} & =\boldsymbol{G} \boldsymbol{P}_{0}+\boldsymbol{P}_{0} \boldsymbol{P}_{1}: \\
\boldsymbol{G} \boldsymbol{P}_{1} & =\frac{\boldsymbol{R}^{g i} \times \boldsymbol{M}_{G}^{g i}}{R^{g i^{2}}}-\frac{\left(\boldsymbol{R}^{g i} \cdot \boldsymbol{M}_{G}^{g i}\right) R^{g i} \times \boldsymbol{n}}{\left(\boldsymbol{R}^{g i} \cdot \boldsymbol{n}\right) R^{g i^{2}}}+\lambda \boldsymbol{R}^{g i} \\
\forall \lambda & \in \Re .
\end{aligned}
$$

Fig. 6 shows the ground $\Pi$, the CoM $G$, the central axis $\left(\Delta_{0}\right)$, and the noncentral axis $\left(\Delta_{1}\right)$ characterized by the ground normal vector $\boldsymbol{n}$. The ZMP $C$ is the intersection point between the axis $\left(\Delta_{1}\right)$ and the ground surface $\Pi$. The ZMP is not the projection of CoM along $\boldsymbol{R}^{g i}$. 


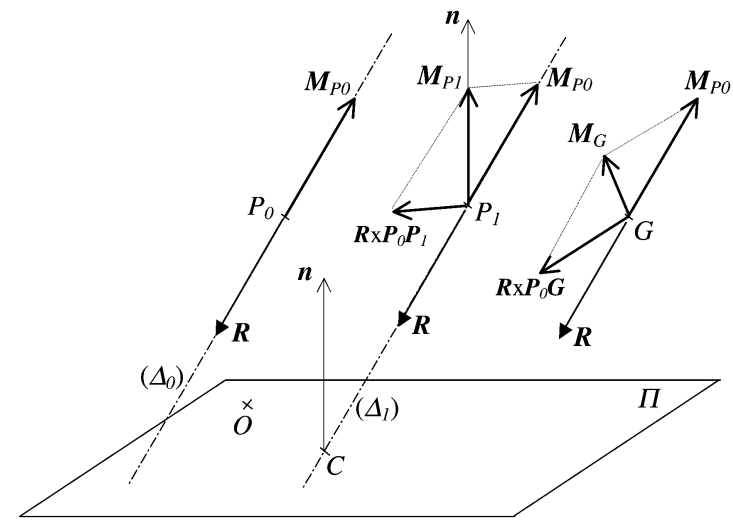

Fig. 6. ZMP location $C$ with respect to the $\operatorname{CoM} G: C$ is not the projection of $G$ along $\boldsymbol{R}$ ( $\boldsymbol{R}$ stands for $\boldsymbol{R}^{g i}$ and $M$ for $\left.M^{g i}\right)$.

\section{APPENDIX II}

\section{A. Developed Expressions of Gravity Plus Inertia Terms}

Equations (8) and (9) are computed with sums such as

$$
\begin{aligned}
\boldsymbol{R}^{g i} & =m \boldsymbol{g}-\sum_{i=1, n} m_{i} \boldsymbol{a}_{G_{i}} \\
\boldsymbol{M}_{Q}^{g i} & =\sum_{i=1, n}\left[m_{i} \boldsymbol{Q} \boldsymbol{G}_{i} \times\left(\boldsymbol{g}-\boldsymbol{a}_{G_{i}}\right)-\dot{\boldsymbol{H}}_{G_{i}}\right]
\end{aligned}
$$

where $m_{i}$ is the mass of the link $L_{i}, G_{i}$ is its CoM, $\boldsymbol{a}_{G i}$ is the acceleration of $G_{i}, \dot{\boldsymbol{H}}_{G i}$ is the rate of angular momentum at point $G_{i}$, and $n$ is the total number of links of the biped.

The rate of angular momentum at point $G_{i}$ is defined as

$$
\dot{\boldsymbol{H}}_{G_{i}}=\boldsymbol{R}_{i}\left(\boldsymbol{I}_{G_{i}} \dot{\boldsymbol{\omega}}_{i}-\left(\boldsymbol{I}_{G_{i}} \boldsymbol{\omega}_{i}\right) \times \boldsymbol{\omega}_{i}\right)
$$

where $\boldsymbol{R}_{i}$ is the rotation matrix associated to the $i$ th solid, $\boldsymbol{I}_{i}$ its inertia matrix, $\boldsymbol{\omega}_{i}$ its rate of rotation, and $\dot{\boldsymbol{\omega}}_{i}$ its angular acceleration.

\section{B. ZMP Computation}

Equation (21) is a summarized expression of scalar formulations often met in literature in order to define the ZMP, which are

$$
\begin{aligned}
x_{D} & =\frac{m g x_{G}-\dot{H}_{O y}}{m\left(g+\ddot{z}_{G}\right)} \\
& =\frac{\sum_{i=1, n}\left(m_{i} x_{G_{i}} g-\left(\dot{H}_{G_{i} y}+m_{i}\left(z_{G_{i}} \ddot{x}_{G_{i}}-x_{G_{i}} \ddot{z}_{G_{i}}\right)\right)\right)}{m g+\sum_{i=1, n} m_{i} \ddot{z}_{G_{i}}} \\
y_{D} & =\frac{m g y_{G}+\dot{H}_{O x}}{m\left(g+\ddot{z}_{G}\right)} \\
& =\frac{\sum_{i=1, n}\left(m_{i} y_{G_{i}} g+\left(\dot{H}_{G_{i} x}+m_{i}\left(y_{G_{i}} \ddot{z}_{G_{i}}-z_{G_{i}} \ddot{y}_{G_{i}}\right)\right)\right)}{m g+\sum_{i=1, n} m_{i} \ddot{z}_{G_{i}}} .
\end{aligned}
$$

These expressions are only true if the ground is horizontal, i.e., if the ground surface normal vector is $\boldsymbol{n}=\boldsymbol{z}$ with $\boldsymbol{g}=-g z$. If the contact surface is inclined, then (20) has to be used

$$
O D=\frac{n \times M_{O}^{g i}}{R^{g i} \cdot n}
$$

with

$$
\boldsymbol{M}_{O}^{g i}=\sum_{i=1, n}\left[m_{i} \boldsymbol{O} \boldsymbol{G}_{i} \times\left(\boldsymbol{g}-\boldsymbol{a}_{G_{i}}\right)-\dot{\boldsymbol{H}}_{G_{i}}\right]
$$

and $\boldsymbol{R}^{g i}$ and $\dot{\boldsymbol{H}}_{G_{i}}$ as defined in Appendix II-A.

\section{APPENDIX III}

This appendix develops the expression of the position of the CoP-ZMP $C$ within the virtual surface $\Pi$ defined by the normal vector

$$
\boldsymbol{n}=\alpha_{1} \boldsymbol{n}_{1}+\alpha_{2} \boldsymbol{n}_{2}
$$

All elements refer to Figs. 3 and 4. $O$ is a point of the intersection line between the two contact surfaces $\Pi_{1}$ and $\Pi_{2}$. The frames of the feet are $\left(O_{i}, \boldsymbol{u}_{i}, \boldsymbol{v}_{i}, \boldsymbol{n}_{i}\right)$ (see Fig. 3). As shown in Fig. 4, the frame of $\Pi$ is $(O, \boldsymbol{u}, \boldsymbol{v}, \boldsymbol{n})$, where

$$
\begin{aligned}
& \boldsymbol{u}=\frac{\boldsymbol{n}_{1} \times \boldsymbol{n}_{2}}{\left\|\boldsymbol{n}_{1} \times \boldsymbol{n}_{2}\right\|} \\
& \boldsymbol{v}=\boldsymbol{n} \times \boldsymbol{u} .
\end{aligned}
$$

Each surface $\Pi_{i}$ can be defined by $\left(O, \boldsymbol{u}, \boldsymbol{y}_{i}, \boldsymbol{n}_{i}\right)$, where

$$
\boldsymbol{y}_{i}=\boldsymbol{n}_{i} \times \boldsymbol{u} .
$$

Let us consider the points $A_{1}$ and $A_{2}$, the normal projections of $C_{1}$ and $C_{2}$ on the line $(O, \boldsymbol{u})$, as shown in Fig. 4

$$
\begin{aligned}
& \boldsymbol{O \boldsymbol { C } _ { 1 }}=O A_{1} \boldsymbol{u}+A_{1} C_{1} \boldsymbol{y}_{1} \\
& \boldsymbol{O \boldsymbol { C } _ { 2 }}=O A_{2} \boldsymbol{u}+A_{2} C_{2} \boldsymbol{y}_{2} .
\end{aligned}
$$

Let us decompose $\boldsymbol{O C}$ in an analog manner with a point $A_{3}$

$$
\boldsymbol{O C}=O A_{3} \boldsymbol{u}+A_{3} C \boldsymbol{v} .
$$

The expression of $\boldsymbol{O C}$ must be computed from (27), the fundamental equation defining the CoP-ZMP

$$
O C=\frac{\boldsymbol{n} \times \boldsymbol{M}_{O}^{c}}{\boldsymbol{R}^{c} \cdot \boldsymbol{n}}
$$

where $\boldsymbol{R}^{c}$ and $\boldsymbol{M}_{O}^{c}$ are the resultant force and moment from the two feet, as expressed in Section IV-B through (28) and (29).

Let $\theta=\operatorname{angle}\left(\boldsymbol{y}_{1}, \boldsymbol{y}_{2}\right)=\operatorname{angle}\left(\boldsymbol{n}_{1}, \boldsymbol{n}_{2}\right)$.

One gets

$$
A_{3} C=\left(\frac{1}{\boldsymbol{R}^{c} \cdot \boldsymbol{n}}\right)\left(A_{1} C_{1} \cdot R_{1}^{p}+A_{2} C_{2} \cdot R_{2}^{p}\right)
$$

and

$$
\begin{aligned}
O A_{3}= & \left(\frac{1}{\boldsymbol{R}^{c} \cdot \boldsymbol{n}}\right) \\
& \cdot\left[O A_{1} \cdot R_{1}^{p}\left(\alpha_{1}+\alpha_{2} \cos \theta\right)\right. \\
& +O A_{2} \cdot R_{2}^{p}\left(\alpha_{2}+\alpha_{1} \cos \theta\right) \\
& -\left(O A_{1} \cdot R_{y_{1}}^{f}+A_{1} C_{1} \cdot R_{u}^{f}\right) \alpha_{2} \sin \theta \\
& +\left(O A_{2} \cdot R_{y_{2}}^{f}+A_{2} C_{2} \cdot R_{u}^{f}\right) \alpha_{1} \sin \theta \\
& \left.+\left(\alpha_{1} M_{C_{2}}^{f}-\alpha_{2} M_{C_{1}}^{f}\right) \sin \theta\right]
\end{aligned}
$$

with

$$
\begin{aligned}
\boldsymbol{R}^{c} \cdot \boldsymbol{n}= & R_{1}^{p}\left(\alpha_{1}+\alpha_{2} \cos \theta\right) \\
& +R_{2}^{p}\left(\alpha_{2}+\alpha_{1} \cos \theta\right) \\
& +\left(\alpha_{1} R_{y_{2}}^{f}-\alpha_{2} R_{y_{1}}^{f}\right) \sin \theta .
\end{aligned}
$$


Equations (i) and (k) demonstrate that the single case where one can get $A_{3} C=\alpha_{1} \cdot A_{1} C_{1}+\alpha_{2} \cdot A_{2} C_{2}$ exists when

$$
\begin{aligned}
& \alpha_{1}=\frac{R_{1}^{p}}{\left\|R_{1}^{p} \boldsymbol{n}_{1}+R_{1}^{p} \boldsymbol{n}_{2}\right\|} \\
& \alpha_{2}=\frac{R_{2}^{p}}{\left\|R_{1}^{p} \boldsymbol{n}_{1}+R_{2}^{p} \boldsymbol{n}_{2}\right\|}
\end{aligned}
$$

and under the condition that the friction tangential forces $R^{f}$ vanish. So, one can consider the above expressions of $\alpha_{1}$ and $\alpha_{2}$ as the best. Concerning $O A_{3}$, expressing the position of the CoP-ZMP along vector $\boldsymbol{u},(\mathrm{j})$ and (k) demonstrate that one never gets

$$
O A_{3}=\alpha_{1} \cdot O A_{1}+\alpha_{2} \cdot O A_{2}
$$

A last remark reinforces the choice we propose for $\alpha_{1}$ and $\alpha_{2}$. Indeed, in the two-dimensional (2-D) case (when $O A_{1}=O A_{2}$ ), this is the single solution for which the pseudo-CoP-ZMP $C$ is inside the 2-D plane (the single solution for which $O A_{3}=$ $O A_{1}=O A_{2}$ ), when moreover the friction contact forces are zero. For any other choice, the virtual CoP-ZMP is outside the plane where the 2-D biped is represented.

\section{REFERENCES}

[1] P. Sardain, M. Rostami, and G. Bessonnet, "An anthropomorphic biped robot: Dynamic concepts and technological design," IEEE Trans. Syst. Man Cybern. A, vol. 28, pp. 823-838, Nov. 1998.

[2] B. Espiau and P. Sardain, "The antropomorphic biped robot BIP2000," in Proc. IEEE Int. Conf. Robot. Automation, San Francisco, CA, 2000, pp. 3997-4002.

[3] C. Azevedo, "Control architecture and algorithms of the anthropomorphic biped robot Bip2000," in Proc. Int. Conf. Climbing Walking Robots, Madrid, Spain, 2000, pp. 285-293.

[4] P. Sardain and G. Bessonnet, "Gait analysis of a human walker wearing robot feet as shoes," in Proc. IEEE Int. Conf. Robot. Automation, Seoul, Korea, 2001, pp. 2285-2292.

[5] — " "Zero moment point. Measurements from a human walker wearing robot feet as shoes," IEEE Trans. Syst. Man Cybern. A, vol. 34, Sept. 2004, to be published.

[6] M. Vukobratovic and D. Juricic, "Contribution to the synthesis of biped gait,” IEEE Trans. Biomed. Eng., vol. 16, no. 1, pp. 1-6, 1969.

[7] M. Vukobratovic, B. Borovac, D. Surla, and D. Stokic, Scientific Fundamentals of Robotics 7. Biped Locomotion: Dynamics, Stability, Control and Application. Berlin, Germany: Springer-Verlag, 1990.

[8] A. Goswami, "Postural stability of biped robots and the foot rotation indicator point," Int. J. Robot. Res., vol. 18, no. 6, pp. 523-533, 1999.
[9] K. Hirai, M. Hirose, Y. Haikawa, and T. Takenaka, "The development of Honda humanoid robot," in Proc. IEEE Int. Conf. Robot. Automation, Leuven, Belgium, 1998, pp. 1321-1326.

[10] T. Takenaka, T. Hasegawa, and Honda, "Gait Generation System for a Legged Mobile Robot,” US Patent 5357433, 1994.

[11] T. Takenaka, M. Nishikawa, and Honda, "Locomotion Control System for Legged Mobile Robot,” US Patent 5432417, 1995.

[12] T. Takenaka, T. Hasegawa, T. Matsumoto, and Honda, "Leg Type Mobile Robot Control Apparatus," US Patent 6243623, B1, 2001.

[13] E. Garcia, J. Estremera, and P. G. de Santos, "A classification of stability margins for walking robots," in Proc. Int. Symp. Climbing Walking Robots, Paris, France, 2002, pp. 799-808.

[14] M. Vukobratovic, B. Borovac, and D. Surdilovic, "Zero moment point-Proper interpretation and new applications," in Proc. IEEE-RAS Int. Conf. Humanoid Robots, Tokyo, Japan, 2001, pp. 237-244.

[15] P. B. Wieber, "On the stability of walking systems," in Proc. Int. Workshop Humanoid Human Friendly Robotics, Tsukuba, Japan, 2002, pp. 53-59.

[16] K. Harada, S. Kajita, K. Kaneko, and H. Hirukawa, "ZMP analysis for arm/leg coordination," in Proc. IEEE-RSJ Int. Conf. Intel. Robots Syst., Las Vegas, NV, USA, 2003, pp. 75-81.

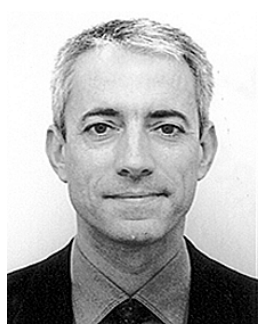

Philippe Sardain received the Professeur agrégé degree from the University of Toulouse, Toulouse, France, in 1984 and the Ph.D. degree in mechanics from the University of Poitiers, Poitiers, France, in 1993.

He taught computer-aided mechanical design at the Ecole Nationale Supérieure de Mécanique et Aérotechnique of Poitiers, from 1985 to 1992. He was in charge of a three-year mission at the Institut National de la Recherche en Informatique et Automatique of Grenoble, France, in the biped robot project "Bip." He is presently Maître de Conférences at the Institute of Technology, University of Poitiers, where he teaches mechanics. In the Laboratoire de Mécanique des Solides of Poitiers, his research interests include robot design, dynamics of robots, and dynamics of human motion.

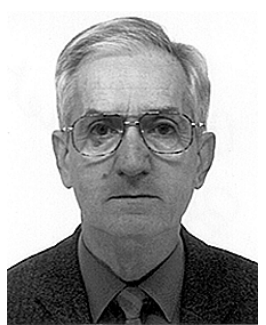

Guy Bessonnet received the M.S. degree in applied mathematics and the Ph.D. degree in fluid mechanics from the University of Poitiers, Poitiers, France, in 1967 and 1971, respectively, and the Doctorat d'Etat ès Sciences Physiques degree in 1991.

$\mathrm{He}$ is a Professeur at the Poitiers University Institute of Technology and a teacher at the Faculté des Sciences, University of Poitiers. He is a Reseacher on the Robotic Team, Laboratoire de Mécanique des Solides of Poitiers. His research interests include optimal motion synthesis of multibody systems, dynamics of robots, dynamics of human motion. 